\title{
Amalthea's modulation of Jovian decametric radio emission
}

\author{
O.V. Arkhypov ${ }^{1}$ and H. O. Rucker ${ }^{2}$ \\ 1 Institute of Radio Astronomy, National Academy of Sciences of Ukraine, Chervonopraporna 4, 61002 Kharkiv, Ukraine \\ e-mail: rai@ira.kharkov.ua \\ 2 Space Research Institute, Austrian Academy of Sciences, Schmidlstrasse 6, 8042 Graz, Austria \\ e-mail: rucker@oeaw.ac.at
}

Received 4 October 2006 / Accepted 9 February 2007

ABSTRACT

\begin{abstract}
Most modulation lanes in dynamic spectra of Jovian decametric emission (DAM) are formed by radiation scattering on field-aligned inhomogeneities in the Io plasma torus. The positions and frequency drift of hundreds of lanes have been measured on the DAM spectra from UFRO archives. A special 3D algorithm is used for localization of field-aligned magnetospheric inhomogeneities by the frequency drift of modulation lanes. It is found that some lanes are formed near the magnetic shell of the satellite Amalthea mainly at longitudes of $123^{\circ} \leq \lambda_{\text {III }} \leq 140^{\circ}$ (north) and $284^{\circ} \leq \lambda_{\text {III }} \leq 305^{\circ}$ (south). These disturbances coincide with regions of plasma compression by the rotating magnetic field of Jupiter. Such modulations are found at other longitudes too $\left(189^{\circ}\right.$ to $\left.236^{\circ}\right)$ with higher sensitivity. Amalthea's plasma torus could be another argument for the ice nature of the satellite, which has a density less than that of water.
\end{abstract}

Key words. planets and satellites: individual: Amalthea - planets and satellites: individual: Jupiter - scattering - plasmas magnetic fields - radiation mechanisms: non-thermal

\section{Introduction}

Amalthea $(270 \times 165 \times 150 \mathrm{~km})$, at an orbit with a $181400 \mathrm{~km}$ radius, is the largest body after the Galilean satellites near Jupiter. An anomaly in Jovian synchrotron radiation has been found on the Amalthea magnetic shell (de Pater et al. 1997). It has been suggested that Amalthea's motion through Jupiter's magnetic field induces Alfven or whistler wings or electrostatic highfrequency waves which lead to pitch angle scattering. We search for another effect of these processes: magnetospheric inhomogeneities, which could be found via scattering of Jovian decametric radio emission (DAM).

Such scattering on field-aligned inhomogeneities in the Io plasma torus is known as "modulation lanes" in dynamical spectra of DAM. Modulation lanes are systems of quasi-periodic and quasi-parallel bands in dynamical spectra of Jovian decametric emission (Fig. 1a). Typically, these bands have a quasi-period of a few seconds and frequency drift of $\sim 100 \mathrm{kHz} / \mathrm{s}$.

Imai et al. (1992, 1997, 2002) have shown that most of the lanes are formed by radiation scattering on field-aligned inhomogeneities in the Io plasma torus. There are strong experimental arguments for the Imai explanation.

(a) Field-aligned inhomogeneities of the Io plasma torus (depleted flux tubes with deep drops of plasma density by about one order of magnitude) were confirmed by measurements in situ with the Galileo probe (Russel et al. 2005; Chust et al. 2005). Their diameters $(\sim 100 \mathrm{~km})$ are about that of the first Fresnel zone in the Io torus: $2 \sqrt{\lambda D} \approx 150 \mathrm{~km}$ with the DAM wavelength of $\lambda \approx 0.015 \mathrm{~km}$ and the source-torus distance of $D \approx 4 \times 10^{5} \mathrm{~km}$. As the effective thickness or essential range of a DAM ray is $\sim 2 \sqrt{\lambda D}$, such filaments are effective modulators of DAM. (b) The Imai model reproduces the slope and curvature of modulation lanes over their whole frequency range $(18-29 \mathrm{MHz}$; Imai et al. 1997, 2002; Arkhipov 2003a).

(c) The calculated variations in drift rate of modulation lanes agree with experimental diagrams depending on the central meridian longitude of Jupiter (Imai et al. 2002; Arkhipov 2003a).

(d) The mean time scale of modulation lane repetition agrees with the diameter of the first Fresnel zone $(2 \sqrt{\lambda D})$ at different distances $D$ from the radio source $\left(0.5 R_{\mathrm{J}}\right.$ and $6 R_{\mathrm{J}}$, where $R_{\mathrm{J}}$ is the equatorial radius of Jupiter; Arkhipov 2004, 2005; Imai et al. 1992, 1997). Therefore, the modulation lanes are indeed of an interference nature.

So far only Imai's model reproduces (b)-(d).

The weak points of Imai's model are:

- the nonrealistic assumption of periodical plasma enhancements only along Io's orbit in the form of equidistant columns being perpendicular to Io's orbital plane;

- failure to explain the spectral patterns with intersecting modulation lanes (Fig. 1a).

To overcome these difficulties, the improved model is used with a more correct algorithm for 3D-localization of field-aligned magnetospheric inhomogeneities by the frequency drift of modulation lanes (Sect. 2). For example, modulation with an opposite sign of frequency drift relative to Io lanes in intersecting spectra (Fig. 1a) was identified with inhomogeneities in the low magnetosphere of Jupiter, near the radio source $\left(D \sim 0.5 R_{\mathrm{J}}\right.$; Arkhipov 2003a, 2004). An analogous technique could be used to search for lane clusters near Amalthea's magnetic shell.

As the lane drift is controlled by the DAM source position too, we localize the source by the average drift of the lanes of an Io torus with known radius (Sect. 3). After this calibration, 


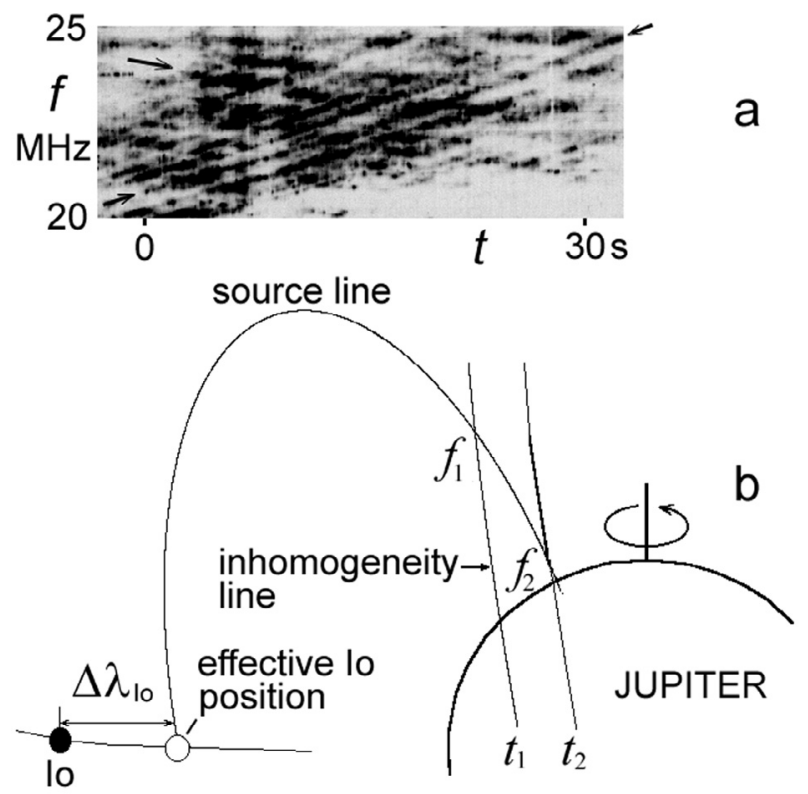

Fig. 1. Modulation lanes in the spectrum of Jovian decametric emission: a) the sample of the radio storm of 08.09.1987 (Riihimaa 1993); b) the scheme of lane formation.

the modulation lane method is used for general localization of magnetospheric inhomogeneities (Sect. 4). Section 5 considers additional observational data with higher sensitivity. The results are summarized in Sect. 6.

\section{Model and algorithms}

We use a more realistic approach than Imai's model.

- No perpendicular (to the equator) inhomogeneities in the Io plasma torus. We track the magnetic lines numerically according to detailed models of the Jovian magnetic field.

- No periodic phase screen occurs in our model. There is rather a chaos of field-aligned inhomogeneities in the region of lane formation like that in the terrestrial polar magnetosphere. The quasi-periodicity (no exact period in general) of modulation lanes are the result of interference fringes from inhomogeneities in a limited layer of the magnetosphere, e.g. the Io torus (Fig. 2).

- Field-aligned magnetospheric inhomogeneities are assumed outside of Io torus, too.

These improvements allow us to overcome the mentioned difficulties emerging from the Imai model. For this purpose, the special 3D algorithm DRIFT has been used to calculate the frequency drift of modulation lanes from a field-aligned magnetospheric inhomogeneity (Arkhipov 2003a).

We employ the so-called standard model for the production of Io-related decametric emission (e.g., Imai et al. 1997) with the discussed improvements. The radio emission is generated on the active magnetic lines, which are activated by the Io satellite (Fig. 1b). The frequency $f$ of each component of the emitted band is approximately equal to the local electron cyclotron frequency at the point of the active line from which it was emitted. Hence, the decametric sources are localized in the polar magnetosphere, at altitudes $\sim 0.1 R_{\mathrm{J}}$, where $R_{\mathrm{J}}=71372 \mathrm{~km}$ is Jupiter's equatorial radius. Io stimulates emission with some time-delay. Accordingly, Io's orbital longitude increases on the lead angle $\Delta \lambda_{\mathrm{Io}}$ in the frame of the Jovian magnetic field during this delay.
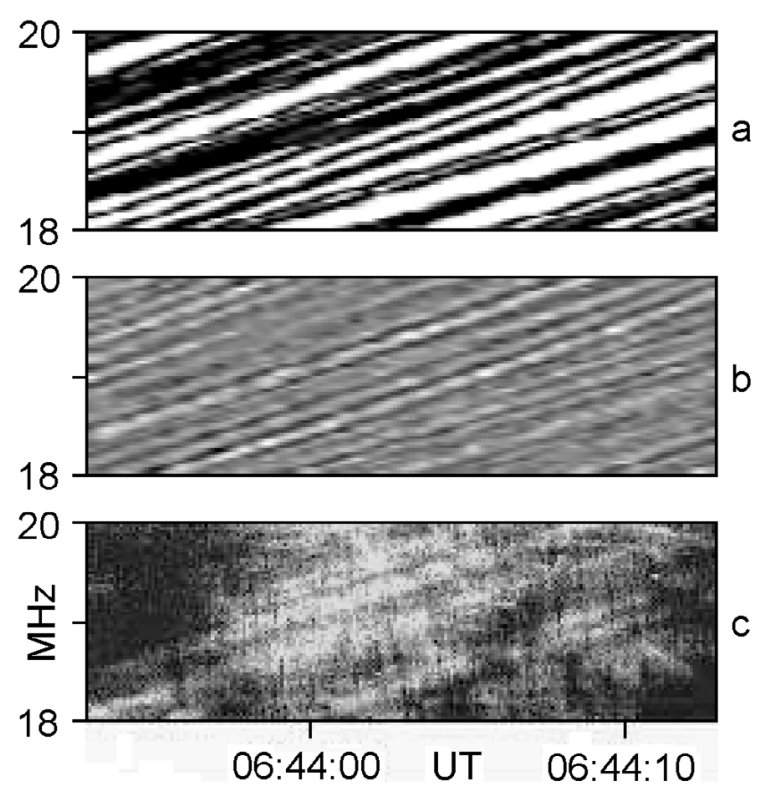

Fig. 2. The numerical simulation of modulation origin: a) the chaotic appearance of field-aligned inhomogeneities of Io plasma torus (the inhomogeneity radius is assumed to be $30 \mathrm{~km}$; the electron number density inside is adopted at the 90 per cent of normal level in the torus which is about $1000 \mathrm{~cm}^{-3}$ ); b) the quasi-periodic pattern of modulation lanes after DAM interference in the phase screen (a) (calculated by the summarizing the wave phases of many photons that cross the phase screen at random points with, the corresponding phase corrections); c) the real lanes in the DAM spectrum of February 16, 2004 (Flagg 2004) with identical Jupiter-Io-observer geometry.

Therefore, the active magnetic line intersects Io's orbit at the point with a longitude of $\lambda_{\text {Io }}-\Delta \lambda_{\text {Io }}$.

The inner magnetosphere co-rotates with the Jovian magnetic field. Accordingly, for the observer, the magnetospheric field-aligned inhomogeneity moves on the background of the active magnetic field (Fig. 1b). The inhomogeneity, as a phase screen, modifies the radiation flux from the active line on the frequency $f_{1}$ with time $t_{1}$. For time $t_{2}$, the projection point corresponds to another radiation frequency $f_{2}$. Thus the drifting modulation lane is formed in the dynamical spectrum of radio emission.

The DRIFT algorithm tracks the magnetic lines with the VIP4 and current sheet models (Connerney et al. 1981, 1998). It searches for the point where the line of inhomogeneity is projected to the magnetic line of the radio source. The frequency of emission at this point could be found as the local gyrofrequency of electrons. Such frequency approximations $f_{1}$ and $f_{2}$ for different times $\left(t_{1}=t-\Delta t / 2, t_{2}=t+\Delta t / 2\right)$ lead to the lane drift estimation: $\mathrm{d} f / \mathrm{d} t=\left(f_{2}-f_{1}\right) / \Delta t$.

The frequency drift of the lane was shown to be dependent the scattering region position in Jovian magnetosphere (Arkhipov 2003a). Accordingly, the DRIFT procedure is a basis for space localization of radio sources or magnetospheric inhomogeneity with other algorithms.

Thus the equality of the calculated $\mathrm{d} f / \mathrm{d} t$ and observed drift can be achieved by variations of:

(a) the lead angle $\Delta \lambda_{\text {Io }}$, if the position of magnetospheric inhomogeneity is known (OPER algorithm); 
(b) the source-inhomogeneity distance $D$ along the Earth direction, if $\Delta \lambda_{\mathrm{Io}}$ is estimated (DL algorithm).

The celestial mechanic formulae (Meeus 1982) are integrated in all algorithms to calculate geometrical parameters for any time. Technical details could be found in the previous publications (Arkhipov 2003a,b).

These algorithms have been successfully applied to studies of DAM sources (Arkhipov 2003b), the structure of the Io plasma torus (Arkhipov 2005) and the low magnetosphere of Jupiter (Arkhipov 2004).

\section{Localization of radio sources}

The most effective lane forming must be near Io's orbit with a mean radius of $5.91 R_{\mathrm{J}}$, where the satellite's volcanoes give the maximal electron density in its plasma torus. Hence, the modulation lanes of Io's torus could be used localise of the radio source.

We use the published dynamical spectra of Io-related Lbursts, which are recorded at 18 to $29 \mathrm{MHz}$ in University of Florida Radio Observatory - UFRO (Flagg 2004; Imai \& Flagg 2005). The resolution of UFRO spectra $(0.1 \mathrm{~s}$ and $30 \mathrm{kHz})$ is sufficient for a modulation lane study. To estimate $\Delta \lambda_{\text {Io }}$, we measured the average $\mathrm{d} f / \mathrm{d} t$ of the Io torus lanes. These estimations were processed with the OPER algorithm to select the optimal lead angles $\left(\Delta \lambda_{\mathrm{Io}}\right)$ for equality of calculated and observed lane drift. The DAM archive of the Nançay Radio Observatory (http://www.obs-nancay.fr/dam/quicklook.php) helps to define northern or southern origin of radio emission by its polarization. The results are summarized in Table 1 .

The standard error of lead angle estimation is found with:

$\sigma_{\lambda}=\sqrt{a_{1}^{2} \sigma_{1}^{2}+a_{2}^{2} \sigma_{2}^{2}+a_{3}^{2} \sigma_{3}^{2}+a_{4}^{2} \sigma_{4}^{2}}$,

where $a_{1}, a_{2}, a_{3}$ are the derivatives of $\Delta \lambda_{\text {Io }}$ with respect to the lane drift, time of observation and mean frequency of radio emission, respectively. $a_{4}$ takes into account the effect of the variations in rotational period of the Io torus due to variable mass loading from Io volcanoes. These partial derivatives have been found numerically with the OPER algorithm by small variations in $\mathrm{d} f / \mathrm{d} t, t, f$ and the angular velocity of the Io torus. $\sigma_{1}, \sigma_{2}$ and $\sigma_{3}$ are standard errors of $\mathrm{d} f / \mathrm{d} t, t$ and $f$ respectively. The typical variation $\sigma_{4}$ in the torus angular velocity is about 3 per cent (Thomas 1993). Obtained $\sigma_{\lambda}$ for different spectra in Table 1 are between 2 and 14 degrees with a mean value of $\left\langle\sigma_{\lambda}\right\rangle=5.6$ degree.

The average estimation of the lead angle is $37 \pm 4^{\circ}$ in accordance with previous results (Arkhipov 2003b, 2004). Apparently, the wide dispersion of $\Delta \lambda_{\text {Io }}$ estimations reflects the variability of the time delay of the interaction between the Io and Jovian low magnetosphere. Such variability has been discussed by other authors (Queinnec \& Zarka 1998; Connerney \& Satoh 2000).

\section{Localization of magnetospheric inhomogeneities}

With $\Delta \lambda_{\text {Io }}$ estimations from Table 1 we can calculate the space position of the formation region for any modulation lane. Hence, the distribution of inhomogeneities in the Jovian magnetosphere could be constructed. The drifts, frequencies and times of 592 modulation lanes were measured on the UFRO spectra listed in Table 1. These data are processed with DL algorithm and with the respective estimates of lead angle (Table 1).
Table 1. Localization of DAM sources with modulation lanes of the Io torus in UFRO spectra.

\begin{tabular}{ccccccc}
\hline \hline $\begin{array}{c}\text { Date } \\
\text { [dd/mm/yy] }\end{array}$ & $\begin{array}{c}\mathrm{UT}^{*} \\
{[\mathrm{hh} \mathrm{mm}]}\end{array}$ & $\begin{array}{c}f^{*} \\
{[\mathrm{MHz}]}\end{array}$ & $\begin{array}{c}\mathrm{d} f / \mathrm{d} t^{*} \\
{[\mathrm{kHz} / \mathrm{s}]}\end{array}$ & $\begin{array}{c}\text { Hemi- } \\
\text { sphere }\end{array}$ & $\begin{array}{c}\Delta \lambda_{\mathrm{Io}} \\
{[\mathrm{deg} .]}\end{array}$ & Ref. \\
\hline $14 / 1 / 2004$ & $09: 32$ & 21.1 & -91.1 & $S$ & $30 \pm 13$ & $\dagger$ \\
$04 / 2 / 2004$ & $08: 58$ & 20.7 & -106.7 & $N$ & $39 \pm 4$ & $\dagger$ \\
$06 / 2 / 2004$ & $10: 13$ & 19.6 & -77.7 & $N$ & 11 & $\dagger$ \\
$15 / 2 / 2004$ & $05: 52$ & 20.2 & -102.8 & $S$ & $52 \pm 3$ & $\dagger$ \\
$16 / 2 / 2004$ & $06: 14$ & 20.5 & 113.3 & $N$ & $34 \pm 3$ & $\dagger$ \\
$22 / 2 / 2004$ & $04: 45$ & 21.1 & -97.8 & $N$ & $50 \pm 14$ & $\dagger$ \\
$23 / 2 / 2004$ & $07: 16$ & 20.2 & 102.2 & $N$ & $35 \pm 5$ & $\dagger$ \\
$29 / 2 / 2004$ & $05: 02$ & 21.2 & -108.4 & $N$ & $28 \pm 5$ & $\dagger$ \\
$25 / 3 / 2004$ & $03: 13$ & 20.7 & -93.4 & $S$ & $44 \pm 6$ & $\dagger$ \\
$26 / 3 / 2004$ & $03: 26$ & 21.1 & 117.2 & $N$ & $39 \pm 3$ & $\dagger$ \\
$02 / 4 / 2004$ & $02: 43$ & 19.6 & 117.3 & $N$ & $57 \pm 4$ & $\dagger$ \\
$09 / 1 / 2005$ & $08: 36$ & 20.1 & -111.3 & $N$ & $5 \pm 5$ & $\dagger$ \\
$17 / 1 / 2005$ & $13: 13$ & 23.3 & 190.4 & $N$ & $56 \pm 2$ & $\ddagger$ \\
$10 / 2 / 2005$ & $07: 13$ & 21.1 & -95.1 & $S$ & $43 \pm 5$ & $\ddagger$ \\
\hline
\end{tabular}

${ }^{*}$ Average for the radio storm. $\dagger$ Flagg (2004). $\$$ Imai \& Flagg (2005).

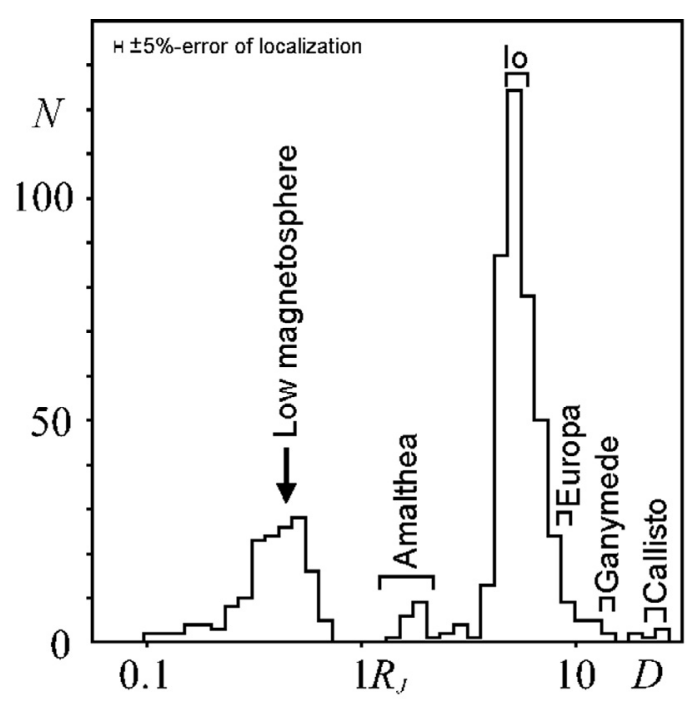

Fig. 3. Distance $D$ between the radio source and the magnetospheric inhomogeneity. $N$ is the number of estimations according to the analysis of the UFRO spectra from Table 1.

Obtained 1D- and 2D-distributions of magnetospheric inhomogeneities are shown in Figs. 3 and 4.

The standard errors of localization could be estimated with the same approach as Eq. (1), but the DL algorithm is used for $a_{1}, a_{2}, a_{3}, a_{4}$ calculations. In addition, the lead angle error and the resolution of UFRO spectra $\left(\sigma_{2}=0.1 \mathrm{~s} ; \sigma_{3} \approx 0.04 \mathrm{MHz}\right)$ are taken into consideration. Because the typical duration of a modulation lane is $\delta t \sim 10 \mathrm{~s}$, the minimal measurable drift is $\sigma_{1}=\sigma_{3} / \delta t=4 \mathrm{kHz} / \mathrm{s}$. Detailed calculations with the parameters $t, f, \mathrm{~d} f / \mathrm{d} t$ of real individual lanes produce typical errors of 5 per cent in the distance between the radio source and the magnetospheric inhomogeneity $(D)$ or in its planetocentric distance $(R)$. These calculations cover a wide range of distances $0.11 R_{\mathrm{J}} \leq D \leq 26 R_{\mathrm{J}}$. Even at shortest distances the localization error is a few per cent in $D$ or 1 per cent in $R$. This is a consequence of the causal relation between modulation drift and relative motion of magnetic lines in the rotating magnetosphere of Jupiter. Apparently, the localization error is up to 20 per cent 


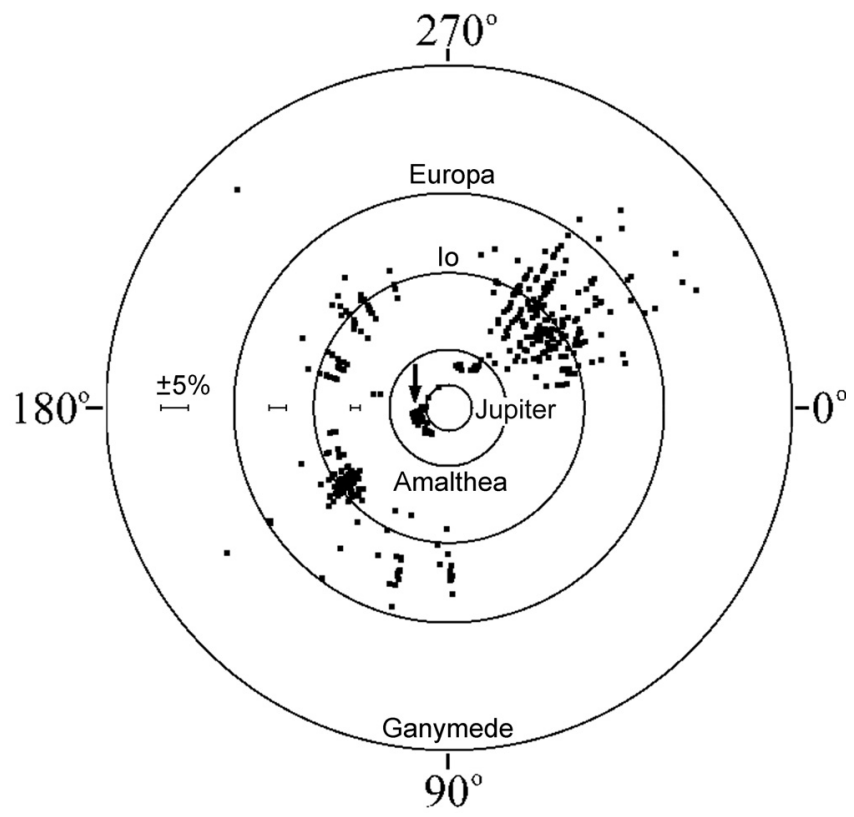

Fig. 4. The distribution of the planetocentric distances $R$ of inhomogeneities (points), which formed lanes at various longitudes (in III 1965 system of Jovian rotation). Horizontal bars show uncertainty in localization with the lane drift error of \pm 5 per cent. The Galilean satellite orbits are shown for comparison. The inhomogeneities of the low magnetosphere are indicated by the arrow.

for some short lanes in the Io-C storm in the longitude sector $300^{\circ}<\lambda<340^{\circ}$ in Fig. 4.

Obviously the majority of lanes concentrate in the Io plasma torus around Io's orbit (peaks at $D \approx R \approx 6 R_{\mathrm{J}}$ in Figs. 3 and 4). However, some lanes are formed around the Europa orbit at $D \approx R \approx 9.4 R_{\mathrm{J}}$. This result argues in favour of Earthbased observability of the Europa torus (Lagg et al. 2003). A few modulation lanes are formally attributed to the Ganymede and Callisto regions (Fig. 3).

Amalthea's orbit coincides with the low peak in Fig. 3 and point clusters in Fig. 4. Such modulation lanes exist as is shown in Fig. 5. Their drift rate is very different from the $\mathrm{d} f / \mathrm{d} t$ of the Io torus lanes. That is why Genova, Aubier \& Lecacheux (1981) described modulation lanes with anomalous low drift rate as a special type of modulation ("high frequency lanes" or HF-lanes), although its nature was unknown at that time. Now we can explain the high frequency of such modulation. The magnetic shell of Amalthea occults radio sources only at low latitudes, usually with $f>20 \mathrm{MHz}$.

Curvatures of HF lanes reflect mainly the decreasing gyromagnetic frequency of electrons with altitude along the magnetic line of the radio source. This is why HF-lanes always have the same curvature. Apparently their association with Io-related storms is a result of an abrupt decrease of non-Io flux and probability with increasing frequency.

The magnetic footprints of magnetospheric inhomogeneities are compactly clustered along the magnetic projection of Amalthea's orbit on the Jovian surface (Fig. 6). Their negligible localization errors $\left(\leq 1^{\circ}\right)$ were found similarly to the method of error estimation for $D$ and $R$ parameters (see above). The modulation lanes are formed at longitudes of $123^{\circ} \leq \lambda_{\text {III }} \leq$ $140^{\circ}$ (north) and $284^{\circ} \leq \lambda_{\text {III }} \leq 305^{\circ}$ (south). These are the longitudes of maximal plasma compression by the rotating magnetic field of Jupiter.

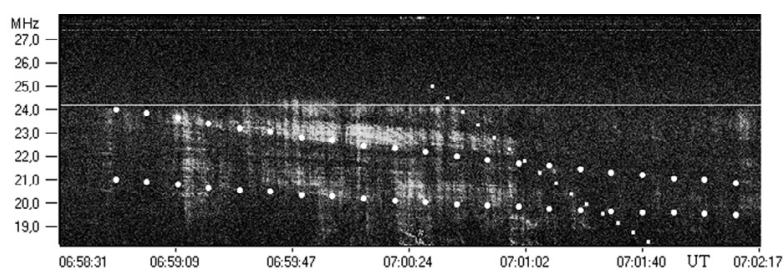

Fig. 5. Amalthea's modulation lanes are seen in the UFRO spectrum of 10.02.2005 (Imai \& Flagg 2005) as slightly inclined bands. The theoretical curves are calculated for the Io magnetic shell (small points) and for the shell with equatorial radius of $207000 \mathrm{~km}$ (bold points). The difference to Amalthea's orbit of 14 per cent can be interpreted in terms of the thickness of Amalthea's torus. For comparison, the adjacent Io torus has a thickness of about half of its radius, up to Europa's orbit.

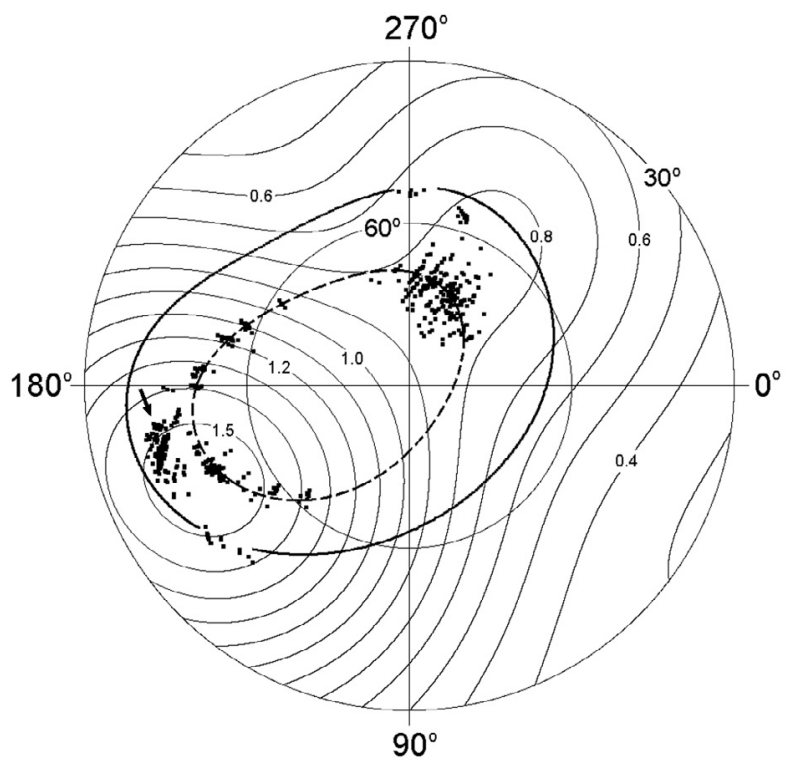

Fig. 6. The magnetic footprints (black squares) of magnetospheric inhomogeneities shown on the map of magnetic strength (marked in $\mathrm{mT}$ ) in the northern Jovian ionosphere. The footprints are calculated for individual modulation lanes on the UFRO spectra from Table 1 . The orbits of Io (dashed oval) and Amalthea (solid oval) are shown with planetographic latitudes and longitudes (III 1965 system). The inhomogeneities of low magnetosphere are indicated by the arrow.

As in the Io torus (Belcher 1987), the freshly ionized material of Amalthea is accelerated from orbital to co-rotational velocities by the Lorentz force of $\boldsymbol{F} \propto[[\boldsymbol{V} \boldsymbol{B}] \boldsymbol{B}]$, where $\boldsymbol{V}$ is the plasma velocity relatively to the local Jovian magnetic field $\boldsymbol{B}$. The main component of this force is azimuthal $F_{\lambda} \propto B^{2}$. Hence, the plasma is compressed where $\partial F_{\lambda} / \partial \lambda_{\text {III }}>0$ or $\partial B^{2} / \partial \lambda_{\text {III }}>0$. According to our calculations with the VIP4 magnetic model (Connerney et al. 1998), the maxima of $\partial B^{2} / \partial \lambda_{\text {III }}$ (i.e. maximal plasma compression) approximately coincide with the regions of modulation lane formation (Fig. 7). Unfortunately, the main maximum of the southern curve at $46^{\circ}$ was not observed on the background of a radio storm.

The negative frequency drift is an identifying characteristic of HF-lanes (Genova et al. 1981). Such a drift is natural for Amalthea's lanes in Io-A and Io-C storms, similarly to the modulation from the Io torus. The negative drift of HF lanes in Io-B storms results from lane formation at short distances from the radio source. The emission ray intersects Amalthea's magnetic shell in two regions separated in space. The nearer (to the radio source) region forms lanes with a slightly negative drift; the 


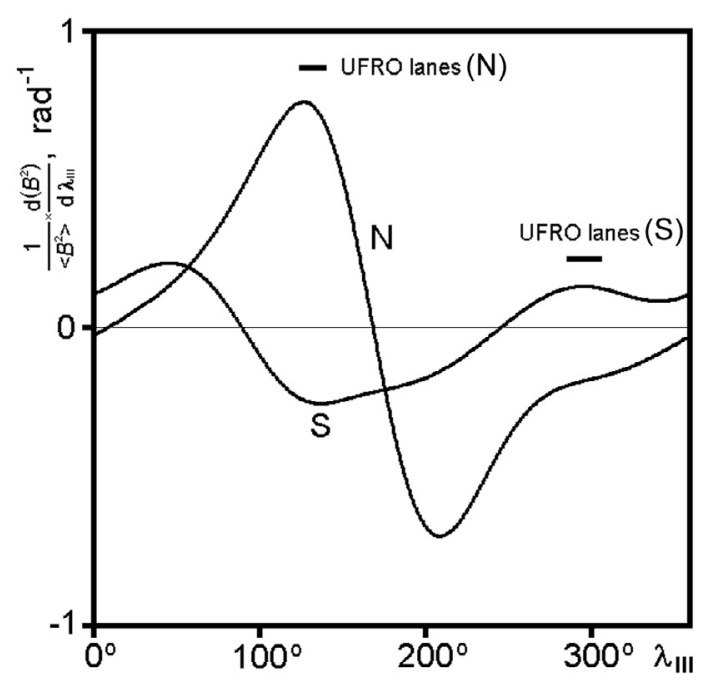

Fig. 7. Compression curves for plasma along Amalthea's orbit at $1 R_{\mathrm{J}}$ above or below the equatorial plane for the occultation of northern or southern radio sources (labeled $\mathrm{N}$ and $\mathrm{S}$, respectively). UFRO modulation lanes formed near Amalthea's shell occupy the longitudinal sectors (marked by horizontal bars) near maximal compression (maxima of the curves). The VIP4 magnetic model (Connerney et al. 1998) has been used here.

farther one forms slightly positive drifts. The narrow radio beam of the northern Io-B storm illuminates the region of compressed plasma predominantly at short distances from the radio source $\left(D \sim 0.5 R_{\mathrm{J}} ; 123^{\circ} \leq \lambda_{\mathrm{III}} \leq 140^{\circ}\right)$. Hence, negative HF drifts dominate in Io-B storms, too.

Another interesting result is the second peak of lane distribution, near the radio source (Fig. 3). Its position at $D_{\max }=0.5 R_{\mathrm{J}}$ corresponds to the altitude of $0.4 R_{\mathrm{J}}$ above the Jovian ionosphere. Thus the modulation lane method gives the unique possibility of studying the low magnetosphere of Jupiter. Supposedly, the deficit of lanes at $D>0.7 R_{\mathrm{J}}$ reflects the decreasing electron number density with altitude.

The average visible distance between inhomogeneities, which are forming modulation lanes in low magnetosphere, is $58 \pm 5 \mathrm{~km}$ (Arkhipov 2004). The typical diameter of the first Fresnel zone is $\left(2 \sqrt{\lambda\langle D\rangle}=44 \mathrm{~km}\right.$ (where $\langle D\rangle=0.5 R_{\mathrm{J}}$; $\lambda=13.3 \mathrm{~m}$ is the wavelength at a typical $f=22.5 \mathrm{MHz}$ ). Hence, modulation lanes are formed by magnetospheric inhomogeneities of about the scale of interference. When this interference scale is less than the monochromatic size of the radio source, modulations are smoothed. This effect can explain the absence of lanes with $D<0.1 R_{\mathrm{J}}$. Therefore, the minimal size of the radio source could be estimated as $\sim 2 \sqrt{\lambda 0.1 R_{\mathrm{J}}}=20 \mathrm{~km}$ with the average $\lambda=14.2 \mathrm{~m}$.

Figure 4 shows that inhomogeneities of the low magnetosphere are concentrated near a longitude of $167^{\circ}$. This cluster is situated on the lines of the known anomaly of the Jovian magnetic field with a maximal magnetic strength (Fig. 6).

\section{Arguments for Amalthea's plasma torus}

To study Amalthea's modulation with more sensitive antennae, we surveyed the Jovian archive of the Space Research Institute of the Austrian Academy of Sciences. Suitable lanes are found on the DAM dynamic spectra of July 6, 1999 (Fig. 8), recorded with the UTR-2 radio telescope at $0.1 \mathrm{~s}$ time resolution (INTAS project 03-51-5727). Two important results were obtained.

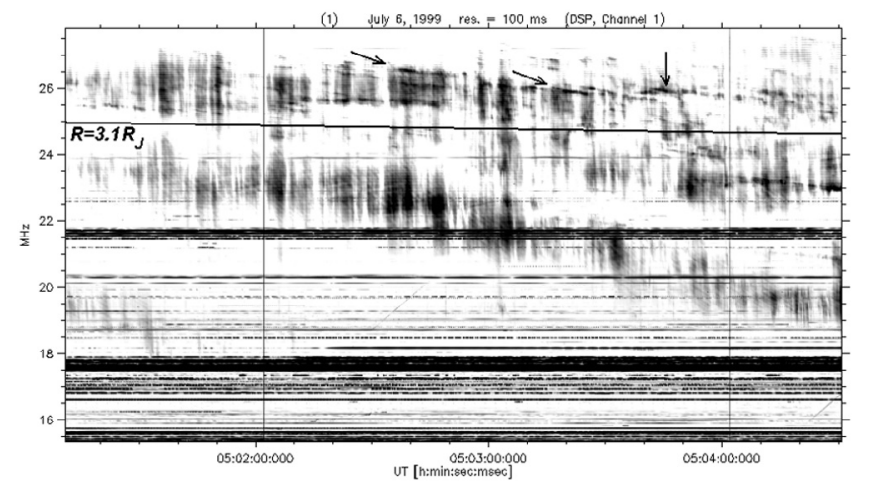

Fig. 8. High frequency lanes (arrowed) in the UTR-2 spectrum of July 6,1999 , although Amalthea's shell $\left(R=2.54 R_{\mathrm{J}}\right)$ did not projected on the radio source. The low frequency border of magnetic shell projection with $R=3.1 R_{\mathrm{J}}$ is shown as a solid line. Another border with $R=3.2 R_{\mathrm{J}}$ coincides with the interference band at $21.6 \mathrm{MHz}$.

Table 2. Parameters of HF-lanes in the UTR-2 spectra of July 6, 1999.

\begin{tabular}{ccccc}
\hline \hline $\begin{array}{c}\text { UT time } \\
{[\mathrm{hh} \mathrm{mm}]}\end{array}$ & $\begin{array}{c}f^{*} \\
{[\mathrm{MHz}]}\end{array}$ & $\begin{array}{c}\mathrm{d} f / \mathrm{d} t^{*} \\
{[\mathrm{kHz} / \mathrm{s}]}\end{array}$ & $\begin{array}{c}R^{*} \\
{\left[R_{\mathrm{J}}\right]}\end{array}$ & $\begin{array}{c}\text { Number } \\
\text { of lanes }\end{array}$ \\
\hline $05: 01-04$ & $25.6 \pm 0.3$ & $-14.6 \pm 1.9$ & $3.14 \pm 0.02$ & 13 \\
$05: 23-26$ & $25.1 \pm 0.4$ & $-18.9 \pm 2.1$ & $3.07 \pm 0.05$ & 11 \\
$06: 05-08$ & $25.0 \pm 0.2$ & $-14.49 \pm 1.5$ & $2.81 \pm 0.04$ & 23 \\
$06: 23-26$ & $24.9 \pm 0.1$ & $-20.2 \pm 1.4$ & $2.73 \pm 0.02$ & 7 \\
\hline
\end{tabular}

* Averaged for all HF-lanes in this time interval.

Firstly, Amalthea's modulation was observed with disturbance longitudes of $189^{\circ}$ to $236^{\circ}$, far from regions of plasma compression $\left(\lambda_{\text {III }} \sim 127^{\circ}\right.$ and $296^{\circ}$; see above). This is an argument for Amalthea's plasma torus.

Secondly, the lanes were formed on the magnetic shell which was slightly shifted from Amalthea's orbit. For each modulation lane we find the center of its formation. Then the magnetic line is tracked from this center to calculate the Jovicentric distance $(R)$ of the line's intersection with the equatorial plane of the planet. Amalthea's orbit has a radius of $R=2.54 R_{\mathrm{J}}$. However, on July 6, 1999, 05:01-5:04 UT, specific lanes appeared with $R=3.14 \pm 0.02 R_{\mathrm{J}}$. Practically the same estimations are obtained with different magnetic field models (O4, O6 and VIP4; Connerney et al. 1998). There are analogous results in other time intervals (Table 2). From Table 2 it follows that the discrepancy of $R^{*}$ with Amalthea's orbit is from $0.19 R_{\mathrm{J}}$ (or 7 per cent) to $0.6 R_{\mathrm{J}}$ (23 per cent) in the equatorial plane. These residuals were unavoidable because of the specific Jovian geometry during UTR-2 observations. Amalthea's shell did not project on the radio source at that time. This blurring of the torus could be interpreted as due to the existence of centrifugally driven cross- $\mathrm{L}$ diffusion of plasma from Amalthea's shell outwards from Jupiter as well as in Io's torus (Froidevaux 1980).

\section{Conclusions}

1. It is found from UFRO data that some modulation lanes are formed near Amalthea's magnetic shell, in two regions $\left(123^{\circ} \leq \lambda_{\mathrm{III}} \leq 140^{\circ}\right.$ for northern radio sources and $284^{\circ} \leq$ $\lambda_{\text {III }} \leq 305^{\circ}$ for southern ones) of maximal plasma compression by the Jovian magnetic field. However, modulation lanes are found at other longitudes with the more sensitive UTR-2 radio telescope. The existence of a plasma torus 
along Amalthea's orbit is supported. Unlike Io, Amalthea has no volcanoes to form a plasma torus. However, the ice sublimation and ice erosion by radiation creates the Europa torus. These mechanisms could work on Amalthea too. The ice nature of Amalthea was argued for independently from Galileo measurements (Anderson et al. 2005).

2. Sometimes modulation lanes are formed near Europa's orbit. Hence, the Europa torus, found during the Cassini mission, is observable by Earth-based radio astronomy.

3 . There are field-aligned inhomogeneities in the low magnetosphere of Jupiter, at altitudes of $\sim 0.4 R_{\mathrm{J}}$. They are concentrated near the field lines of the magnetic anomaly with maximal magnetic strength in the Jovian ionosphere.

4. The modulation lanes are not formed at distances of $D<$ $0.1 R_{\mathrm{J}}$ from the radio source. The simplest explanation is the smoothing of fine interference pattern with too large a source. The minimal dimension of sources is about $20 \mathrm{~km}$.

Acknowledgements. We used the dynamical spectrum of the Jovian radio storm of July 6,1999 , which was recorded by an observation compaign within the frame of the INTAS project 03-51-5727 (A. Lecacheux, H. O. Rucker, A. A. Konovalenko and Yu. Tokarev) with the UTR-2 radio telescope. We thank Dr. Philippe Zarka for fruitful discussions.

\section{References}

Anderson, J. D., Johnson, T. V., Schubert, G., et al. 2005, Science, 308, 1291 Arkhipov, A. V. 2003a, Kinematics and Physics of Celestial Bodies, 19, 124
Arkhipov, A. V. 2003b, Kinematics and Physics of Celestial Bodies, 19, 265 Arkhipov, A. V. 2004, Kinematics and Physics of Celestial Bodies, 20, 252 Arkhipov, A. V. 2005, Kinematics and Physics of Celestial Bodies, 21, 30 Belcher, J. W. 1987, Science, 238, 170

Chust, T., Roux, A., Kurth, W. S., et al. 2005, Planet. Space Sci., 53, 395

Connerney, J. E. P., Acuna, M. H., \& Ness, N. F. 1981, J. Geoph. Res., 86, A10, 8370

Connerney, J. E. P., Acuna, M. H., Ness, N. F., \& Satoh, T. 1998, J. Geoph. Res., 103, 11929

Connerney, J. E. P., \& Satoh, T. 2000, Phil. Trans. R. Soc. Lond., A358, 2471

de Pater, I., Schulz, M., \& Brecht, S. H. 1997, J. Geophys. Res., 102, 22043

Flagg, R. 2004, Radio JOVE Data Archive Display, http: //jovearchive.gsfc.nasa.gov/

Froidevaux, L. 1980, Geophys. Res. Lett., 7, 33

Genova, F., Aubier, H. G., \& Lecacheux, A. 1981, A\&A, 104, 229

Imai, K., \& Flagg, R. 2005, UFRO spectrograph data archive, http://jupiter.kochi-ct.jp/spectrograph/

Imai, K., Wang, L., \& Carr, T. D. 1992, Origin of Jupiter's decametric modulation lanes, in Planetary Radio Emissions III, ed. H. O. Rucker, S. J. Bauer, B. M. Pedersen (Vienna: Austrian Acad. Sc. Press), 69

Imai, K., Wang, L., \& Carr, T. D. 1997, J. Geophys. Res. 102, 7127

Imai, K., Riihimaa, J. J., Reyes, F., \& Carr, T. D. 2002, J. Geophys. Res. 107, 101029

Lagg, A., Krupp, N., Woch, J., \& Williams, D. J. 2003, Geophys. Res. Lett., 30, 10

Meeus, J. 1982, Astronomical formulae for calculators (Richmond: WillmannBell), 168

Queinnec, J., \& Zarka, Ph. 1998, J. Geophys. Res., 103, 26649

Riihimaa, J. J. 1993, Modulation lanes revisited (Oulu: Univ. Oulu), 92

Russel, C. T., Kivelson, M. G., \& Khurana, K. K. 2005, Planet. Space Sci., 53, 937

Thomas, N. 1993, J. Geophys. Res. 98, 18737 